\title{
SPOTting Eyelid Injuries: A Novel Mobile Application for Managing Periocular Injuries
}

\author{
Devi Prasad Mohapatra ${ }^{1}$ Ravi Kumar Chittoria ${ }^{1} \quad$ Frji $^{1} \quad$ Meethale Thiruvoth ${ }^{1} \quad$ Likhitha Reddy $^{1}$
}

1Department of Plastic Surgery, Jawaharlal Institute of Postgraduate Medical Education and Research, Puducherry, India

Indian J Plast Surg 2019;52:358-359

Sir,

Eyelid and periocular trauma present in myriad forms and need specialized management techniques. The diverse pattern of presentation of these injuries can be classified according to the system for periocular trauma (SPOT). ${ }^{1}$ These injuries can be further treated according to the algorithm based on SPOT. However, recall of the comprehensive SPOT classification can be an issue especially in the chaotic emergency set up where patients with such injuries usually present. To precisely address this issue and avoid recall errors while documenting the periocular injury types according to SPOT in the medical case records, we developed a novel SPOT Eye mobile application (app). The SPOT Eye app is available for free downloads in the android mobile application store at present for touchscreen smartphones (https://play.google.com/store/apps/ details?id=com.eye.spot.classification\&hl=en).

The app, once downloaded, can be opened when the physician is presented with a patient with periocular injury. The physician then proceeds forward along the app pages to map
Address for correspondence Devi Prasad Mohapatra, MCh, Department of Plastic Surgery, 4th Floor SSB, Jawaharlal Institute of Postgraduate Medical Education and Research, Puducherry 605006, India (e-mail: devimohapatra1@gmail.com).

the eyelid injury according to the patient presentation. The app has an interactive interface to map eyelid and periocular injuries of each eye according to the existing injury in the patient. A toggle switch present within the app page for each eye is turned on to indicate lacrimal injuries if present. When the injury is mapped according to patient presentation, the app provides an output of the injury on the result page according to SPOT terminology. The attending physician then enters the output in the medical case record of the patient and the patient is treated for the injuries by the reconstructive oculoplastic surgeon. An illustrative case is presented below.

A 30-year-old man who sustained facial injuries in a road traffic accident was referred to us for management of lacerations around the left eye and face ( $\boldsymbol{- F i g .} \mathbf{1}$ a, b). Clinical examination revealed abrasions over the left side malar region and left ala. A laceration over the left periocular region with partial avulsion of lower eyelid from the lateral canthus and loss of lateral one-fourth of the lower eyelid. The

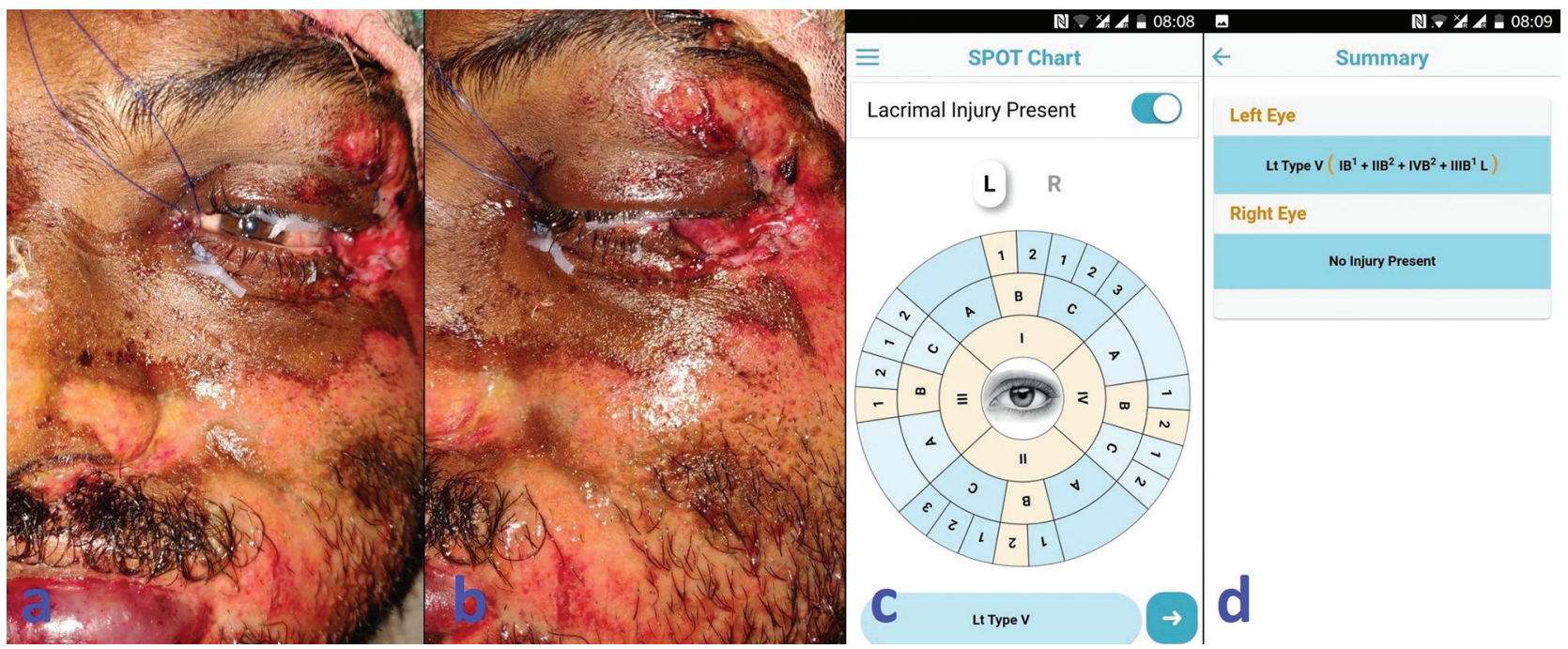

Fig. 1 Periocular laceration left side with (a) eye open and (b) eye closed. (c) Injury mapping on SPOT Eye App. (d) Injury diagnosis output in SPOT Eye app.

received

March 27, 2019

accepted after revision

August 7, 2019

published online

November 28, 2019
DOI https://doi.org/

$10.1055 / \mathrm{s}-0039-3400181$

ISSN 0970-0358.
C2019 Association of Plastic

Surgeons of India
License terms

()(1) $\Theta \circledast$ 


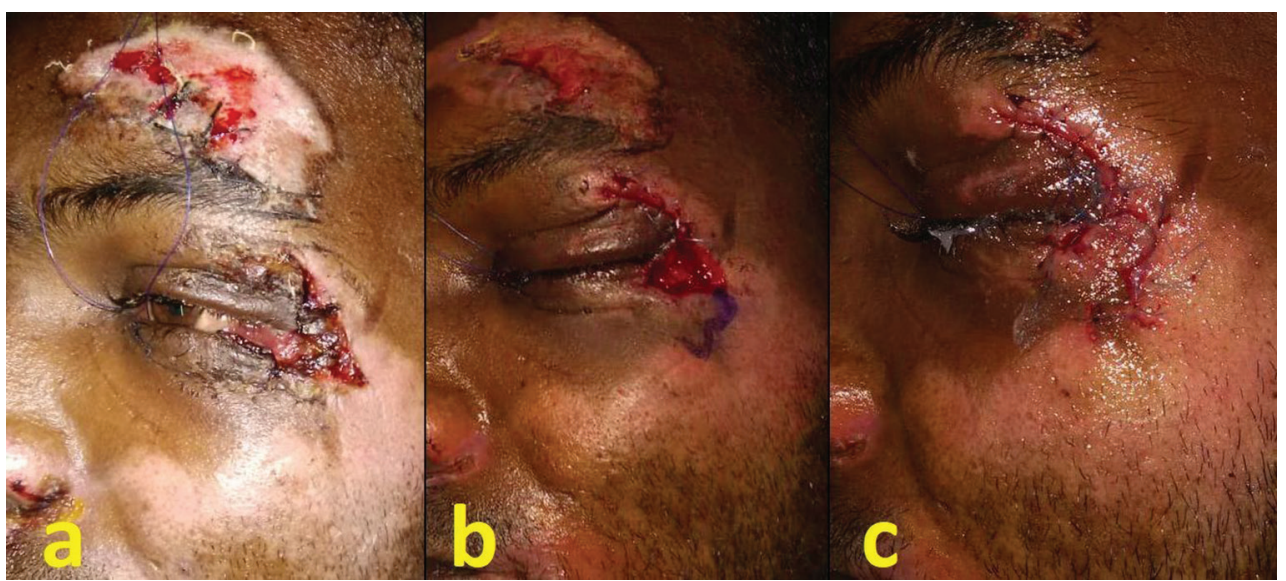

Fig. 2 (a) Healed lower eyelid laceration after 1 week. (b) Bilobed flap marking for lateral lower eyelid loss. (c) Flap in position after 5 days of inset.

laceration extended upward to involve the lateral margin of upper eyelid without loss of tissue in upper eyelid. Medial avulsion of lower eyelid resulted in tearing of the lower lacrimal canaliculi. The SPOT Eye app was used to input the periocular injuries (-Fig. 1c). A diagnosis of Lt Type $\mathrm{V}\left(\mathrm{IB}^{1+}\right.$ $\mathrm{IIB}^{2}+\mathrm{IVB}^{2}+\mathrm{IIIB}^{1} \mathrm{~L}$ ) periocular injury according to SPOT was made ( - Fig. 1d). Primary canalicular and medial lower eyelid laceration repair was done. The lateral lower eyelid and canthal raw area was covered with collagen and planned for delayed repair to assess the final extent of injury. A week later the lower eyelid and canthal region soft tissue reconstruction was done using a bilobed flap ( - Fig. 2). The wounds healed uneventfully. Follow-up at 3 weeks revealed adequate functional and aesthetic outcome ( - Fig. 3 ).

We have been using the SPOT Eye app in our unit for last one year. The app is downloaded by all residents and consultants of our unit and used in management of periocular injuries presenting to our department. With the ubiquity of smartphones and apps, digital technology-based diagnosis and management are being adopted by physicians and surgeons worldwide. ${ }^{2}$ The availability of such a system prevents recall and documentation errors among the attending physicians, especially in trauma units where possibility of documentation errors is high. ${ }^{3,4}$ In addition to this, the SPOT Eye app has the utility in peripheral centers from where

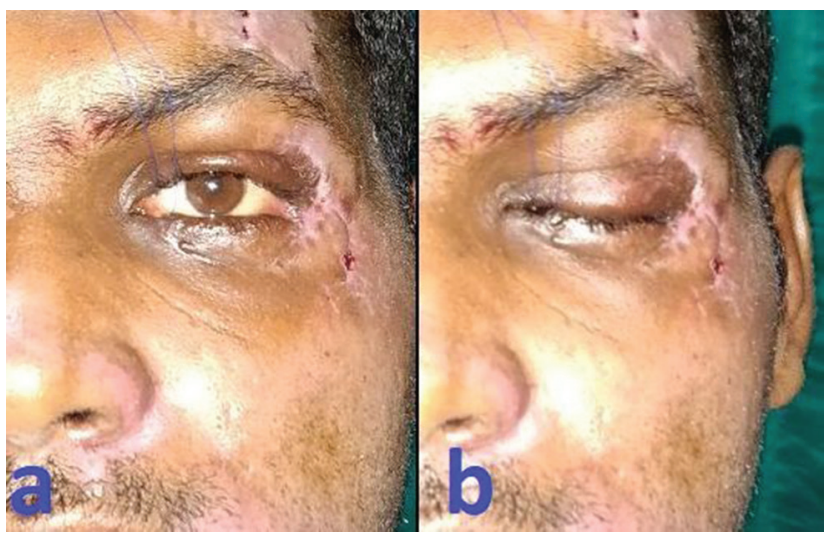

Fig. 3 Healed left periocular injuries at end of 3 weeks. patients with periocular injuries can be referred to higher centres with an accurate diagnosis made by the primary care physician using the app. At present this app is available for android platform, which is a limitation for physicians using Windows and iOS operating system-based smartphones. Some physicians would be uncomfortable with downloading and utilizing new digital technologies. We feel this is a unique app and the concept of use of mobile application as a diagnostic aid can be extended to other reconstructive scenarios as well. We plan to develop a cross-platform app in future. In addition, we plan to provide capability to take pictures within the app itself and also integrate the app with existing hospital information system (HIS).

\section{Source(s) of Support}

None.

\section{Presentation at a Meeting}

Part of this manuscript was presented in the XXVIII annual conference of Oculoplastic Association of India (OPAI), Madurai, On October 27-29, 2017.

\section{Conflict of Interest}

The mobile application presented in the manuscript has been developed by the authors themselves.

\section{Acknowledgment}

None.

\section{References}

1 Mohapatra DP, Thiruvoth FM, Chittoria RK, et al. Proposal of a new classification scheme for periocular injuries. Indian J Plast Surg 2017;50:21-28

2 Mohapatra DP, Mohapatra MM, Chittoria RK, Friji MT, Kumar SD. The scope of mobile devices in health care and medical education. Int J Adv Med Health Res 2015;2:3-8

3 Reusche R, Buchanan PJ, Kozlow JH, Vercler CJ. A systematic review of smartphone applications for plastic surgery providers: target audience, uses, and cost. Ann Plast Surg 2016;77(1):6-12

4 Larson RS. A path to better-quality mHealth apps. JMIR Mhealth Uhealth 2018;6(7):e10414 